\title{
Predictive analyses for balance and gait based on trunk performance using clinical scales in persons with stroke
}

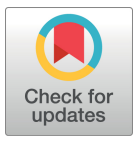

\section{Youngkeun Woo}

Department of Physical Therapy, College of Medical Sciences, Jeonju University, Jeonju, Republic of Korea

Objective: This study aimed to predict balance and gait abilities with the Trunk Impairment scales (TIS) in persons with stroke. Design: Cross-sectional study.

Methods: Sixty-eight participants with stoke were assessed with the TIS, Berg Balance scale (BBS), and Functional Gait Assessment (FGA) by a therapist. To describe of general characteristics, we used descriptive and frequency analyses, and the TIS was used as a predictive variable to determine the BBS. In the simple regression analysis, the TIS was used as a predictive variable for the BBS and FGA, and the TIS and BBS were used as predictive variables to determine the FGA in multiple regression analysis.

Results: In the group with a BBS score of $>45$ for regression equation for predicting BBS score using TIS score, the coefficient of determination $\left(\mathrm{R}^{2}\right)$ was 0.234 , and the $\mathrm{R}^{2}$ was 0.500 in the group with a BBS score of $\leq 45$. In the group with an FGA score $>15$ for regression equation for predicting FGA score using TIS score, the $\mathrm{R}^{2}$ was 0.193 , and regression equation for predicting FGA score using TIS score, the $\mathrm{R}^{2}$ was 0.181 in the group of FGA score $\leq 15$. In the group of FGA score $>15$ for regression equation for predicting FGA score using TIS and BBS score, the $\mathrm{R}^{2}$ was 0.327 . In the group of FGA score $\leq 15$ for regression equation for predicting FGA score using TIS and BBS score, the $\mathrm{R}^{2}$ was 0.316 .

Conclusions: The TIS scores are insufficient in predicting the FGA and BBS scores in those with higher balance ability, and the BBS and TIS could be used for predicting variables for FGA. However, TIS is a strong predictive variable for persons with stroke who have poor balance ability.

Key Words: Gait, Regression analysis, Stroke

\section{Introduction}

The trunk plays an important role in functional independence as well as basic motor control in activity of daily living [1]. However, the recovery or rehabilitation of the trunk is a more neglected area in stroke rehabilitation research or intervention compared to limb rehabilitation [2]. Although persons with have unilateral impairments on one side of the body, most persons with stroke have impaired trunk strength or functional performance [3]. Stroke survivors have decreased control of trunk movements, and these impaired movements of trunk also affect the body's ability to maintain balance and perform rotational movement of the lower parts during walking. Therefore, the trunk should be highly considered when developing rehabilitation goals and for activity of daily living [4].

The trunk is an essential and fundamental component for overall functional independence [5]. The trunk has a strong relationship with functional outcomes such as gait [6], sitting balance [7], and there is a strong positive correlation between trunk performance and balance in stroke [8]. Therefore, it is important to estimate of trunk performance that essential for daily activities of livings and higher performance motoric tasks [8]. However, although trunk performance is

Received: 27 February, 2018 Revised: 14 March, 2018 Accepted: 15 March, 2018

Corresponding author: Youngkeun Woo (ORCID http://orcid.org/0000-0003-4360-7390)

Department of Physical Therapy, College of Medical Sciences, Jeonju University, 303 Cheonjam-ro, Wansan-gu, Jeonju 55069, Republic of Korea Tel: 82-63-220-3163 Fax: 82-63-220-2054 E-mail: ykwoo92@naver.com

(c) This is an Open-Access article distributed under the terms of the Creative Commons Attribution Non-Commercial License (http://creativecommons.org/licenses/ by-nc/4.0) which permits unrestricted non-commercial use, distribution, and reproduction in any medium, provided the original work is properly cited.

Copyright @ 2018 Korean Academy of Physical Therapy Rehabilitation Science 
an important component for predicting balance and functional task, the supporting evidence is not fully suggested in the rehabilitation for the stroke population [3]. Verheyden et al. [7] reported that trunk control is important and an early predictor for activities of daily living in those affected by stroke, and Likhi et al. [5] also reported that trunk impairment is an important predictor for functional independence rather than upper and lower function in stroke. In addition, Kim et al. [9] suggested that trunk performance was not an integral component for predicting functional outcomes in persons with stroke.

Based on previous studies, the relationship between trunk performance and functional task in stroke is a special area of interest for early trunk exercise and improving balancing activities for later stages of rehabilitation [8]. Verheyden et al. [2] mentioned that measuring trunk performance is important for predictive information in stroke survivors. Evaluating trunk performance is measured in various ways, which include the hand held dynamometer, isokinetic dynamometer, posturography and surface electromyography to quantifying trunk performance [3]. Also, a variety of tools for measuring trunk performance is acceptable in clinics with standardized tools [2]. Clinical scales provide a simple and convenient method, numerical values, and also reduce the time spent during assessment in persons affected by stroke $[10,11]$. Early detection is important for preventing fall injury by screening functional tasks as well as any other impairments or complication during stroke rehabilitation $[4,12]$.

Despite the strong and significant relationship between trunk performance with balance and gait, but there is insufficient information on predicting balance and gait based on trunk performance rather than overall activities of daily living. Relationship is performed by correlation statics for describing two variables for strength of relationship, but for predicting one variable by one variable bases on relationship is regression analysis is commonly used [13]. For these reasons, the purpose of this study was to analyze the prediction and explanation of and the relationship between trunk with balance and gait in persons with stroke.

\section{Methods}

\section{Participants}

Sixty-eight persons with stroke participated in this study. Data were collected from both inpatient and outpatient physical therapy rehabilitation facilities in rehabilitation hos- pitals. The general subject characteristics can be viewed in Table 1.

The participants were included if they had been diagnosed with stroke, were able to communicate with the evaluator, and were able to stand without assistance. Participants were excluded if there was a history of neurological, orthopedic, or psychological disorders that would affect their balance and gait, or any cognitive deficits that would impair the ability to comprehend the study procedures. Prior to participating in the study, the subjects provided their informed consent based on the Declaration of Helsinki principles.

\section{Clinical scales}

To investigate the relationship of trunk performance on balance and gait in persons with stroke, three common clinical scales, such as the Trunk Impairment scale (TIS), Berg Balance scale (BBS), and Functional Gait Assessment (FGA), were used for evaluation in a random order by a therapist. Subjects were verbally given standardized instructions in regards to the clinical scales and performed the tests in a quiet room. Rest intervals were provided between each test. Subjects wore their own shoes throughout the evaluation.

The TIS was developed to assess static and dynamic balance and also coordination of the trunk by observation of the quality of trunk performance that affect the performance of activity of daily living in stroke survivors [14]. Also, the TIS was developed to predict the ability of outcomes as well as mobility after stroke. The TIS were assessed on the table and chair, and it is consisted of 17 items with 7 points for static components, 10 points for dynamic components and 6 points for coordinative components. The duration of the assess-

Table 1. General characteristics of the participants of this study

$(\mathrm{N}=68)$

\begin{tabular}{lc}
\hline \multicolumn{1}{c}{ Characteristic } & Value \\
\hline Age $(\mathrm{y})$ & $55.47(11.83)$ \\
Height $(\mathrm{cm})$ & $164.74(8.73)$ \\
Weight $(\mathrm{kg})$ & $63.51(13.91)$ \\
Post-stroke duration (mo) & $22.04(12.13)$ \\
Trunk Impairment scale (scores) & $14.54(5.06)$ \\
Berg Balance scale (scores) & $44.15(9.19)$ \\
Functional Gait Assessment (scores) & $17.91(7.48)$ \\
Gender (male/female) & $41 / 27$ \\
Affected side (left/right) & $42 / 26$ \\
Type (infarction/hemorrhage) & $32 / 36$ \\
\hline
\end{tabular}

Values are presented as mean (SD) or number only. 
ment was 15 minutes, and 23 points indicated a high score. The TIS has a high reliability $(\mathrm{r}=0.98)$ and validity $(\mathrm{r}=0.99)$ $[15,16]$.

The BBS is currently the most common used balance assessment tool related to activity of daily living in the clinic and research area $[9,17]$. The BBS was developed by Berg et al. [18] in 1992. The BBS can be used for discrimination and prediction of a person who may have a high fall risk. It consists of sitting, standing, and changing positions with 14 items. The duration of the BBS is approximately 15 minutes and uses a 5 point scale for evaluation with a maximum score of 56. If the subjects had a score below 45 , the subjects were categorized into the high fall risk group. It was originally developed for detecting fall risk in the elderly population, but it can be also be used persons affected by stroke, traumatic brain injury, cerebral palsy, and Parkinson disease. BBS has a high intra-rater reliability $(\mathrm{r}=0.97)$, and inter-rater reliability $(\mathrm{r}=0.98)$ [18-20].

The FGA has the best discriminative ability in detecting high walking function in persons with stroke [21]. The FGA was developed by Wrisley et al. [22] in 2004 and is used to detect postural stability during variable walking tasks. The FGA was modified reliability and reduced ceiling effect of Dynamic Gait index (DGI), it has 7 items from the DGI, and 3 new items added in FGA. To reduce the ceiling effect of the DGI and to modify its reliability, the FGA was created and includes 7 items from the DGI and three new items. The FGA has a duration of 5 to 10 minutes, with a maximum score of 30 , and a high intra-rater reliability $(\mathrm{r}=0.97)$, and high inter-rater reliability $(\mathrm{r}=0.94)[21,22]$.

\section{Data analysis and statistics}

Descriptive analysis was performed for the subject characteristics, such as age, height, weight, length of time after the stroke incident, TIS, BBS, and FGA scores. The frequency analysis was performed for gender, the side where the paraplegia or paresis was present, and disease type. To determine the relationship between trunk performance and balance and gait abilities based on clinical scales, the simple regression analysis was conducted. The multiple regression analysis was performed to investigate the relationship between variables, with the dependent variables being FGA, and the predictor variables being the TIS and BBS. A significant level of $\alpha<0.5$ was used. Data analysis was performed using IBM SPSS Statistics ver. 23.0 (IBM Co., Armonk, NY, USA).

\section{Results}

\section{Simple regression analyses using the TIS for predicting BBS and FGA}

The simple regression equation was used for calculating the predicted BBS (Table 2) and FGA (Table 3) scores by using the TIS scores. For the regression equation for predicting BBS score using the TIS score, the correlation coefficient (r) was 0.781 , the coefficient of determination $\left(R^{2}\right)$ was 0.610 , the regression constant was 23.523, and the regression coefficient for the BBS score was 1.418. In the group with a

Table 2. The equations for TIS on the BBS by simple regression analysis

\begin{tabular}{lccccc}
\hline Clinical scale & Regression equation & $\mathrm{r}$ & $\mathrm{R}^{2}$ & $\beta^{\text {a }}$ & $\mathrm{F}(p)$ \\
\hline BBS (scores) & $(23.523)+(1.418 \times \mathrm{TIS})$ & 0.781 & 0.610 & 0.781 & $103.090(<0.001)$ \\
BBS $>45\left(\mathrm{n}_{1}=35\right)$ & $(45.673)+(0.444 \times \mathrm{TIS})$ & 0.483 & 0.234 & 0.483 & $10.050(0.003)$ \\
BBS $\leq 45\left(\mathrm{n}_{2}=33\right)$ & $(22.794)+(1.204 \times \mathrm{TIS})$ & 0.707 & 0.500 & 0.707 & $30.997(<0.001)$ \\
\hline
\end{tabular}

TIS: Trunk Impairment scale, BBS: Berg Balance scale.

${ }^{\mathrm{a}}$ Standardized coefficient $(\beta)$.

Table 3. The equations for TIS on the FGA by simple regression analysis

$(\mathrm{N}=68)$

\begin{tabular}{lrrrrc}
\hline Clinical scale & Regression equation & $\mathrm{r}$ & $\mathrm{R}^{2}$ & $\beta^{\mathrm{a}}$ & $\mathrm{F}(p)$ \\
\hline FGA $($ scores $)$ & $(2.149)+(1.084 \times$ TIS $)$ & 0.733 & 0.537 & 0.733 & $76.640(<0.001)$ \\
FGA $>15\left(\mathrm{n}_{1}=37\right)$ & $(15.987)+(0.445 \times$ TIS $)$ & 0.439 & 0.193 & 0.439 & $8.362(0.007)$ \\
FGA $\leq 15\left(\mathrm{n}_{2}=31\right)$ & $(6.710)+(0.381 \times$ TIS $)$ & 0.425 & 0.181 & 0.425 & $6.397(0.017)$ \\
\hline
\end{tabular}

TIS: Trunk Impairment scale, FGA: Functional Gait Assessment.

${ }^{a}$ Standardized coefficient $(\beta)$. 
Table 4. The equations for TIS and BBS on the FGA by multiple regression analysis

$(\mathrm{N}=68)$

\begin{tabular}{lccrr}
\hline Clinical scale & Regression equation & $\mathrm{r}$ & $\mathrm{R}^{2}$ & $p$-value \\
\hline FGA $($ scores $)$ & $-8.864+(0.420 \times$ TIS $)+(0.468 \times \mathrm{BBS})$ & 0.816 & 0.666 & $<0.001$ \\
FGA $>15\left(\mathrm{n}_{1}=37\right)$ & $3.217+(0.340 \times \mathrm{BBS})$ & 0.572 & 0.327 & 0.001 \\
FGA $\leq 15\left(\mathrm{n}_{2}=31\right)$ & $2.824+(0.199 \times \mathrm{BBS})$ & 0.562 & 0.316 & 0.005 \\
\hline
\end{tabular}

TIS: Trunk Impairment scale, BBS: Berg Balance scale, FGA: Functional Gait Assessment.

BBS score of $>45$ for regression equation for predicting the BBS score using the TIS score, the correlation coefficient $(\mathrm{r})$ was 0.483 , the coefficient of determination $\left(R^{2}\right)$ was 0.234 , the regression constant was 45.673 , and the regression coefficient for the BBS score was 0.444. In the group with a BBS score of $\leq 45$ for regression equation for predicting BBS score using TIS score, the correlation coefficient (r) was 0.707 , the coefficient of determination $\left(R^{2}\right)$ was 0.500 , the regression constant was 22.794, and the regression coefficient for the BBS score was 1.204.

For the regression equation for predicting the FGA score using the TIS score, the correlation coefficient (r) was 0.733 , the coefficient of determination $\left(\mathrm{R}^{2}\right)$ was 0.537 , the regression constant was 2.149, and the regression coefficient for the FGA score was 1.084. In the group with an FGA score of $>15$ for regression equation for predicting the FGA score using the TIS score, the correlation coefficient ( $\mathrm{r}$ ) was 0.439 , the coefficient of determination $\left(\mathrm{R}^{2}\right)$ was 0.193 , the regression constant was 15.987 , and the regression coefficient for the FGA score was 0.445 . In the group with an FGA score of $\leq 15$ for regression equation for predicting the FGA score using the TIS score, the correlation coefficient (r) was 0.425 , the coefficient of determination $\left(R^{2}\right)$ was 0.181 , the regression constant was 6.710 , and the regression coefficient for the BBS score was 0.381 .

\section{Multiple regression analysis using the TIS and BBS for predicting FGA}

Table 4 shows the multiple regression equation for calculating the predicted FGA score by using the TIS and BBS score. For the regression equation for predicting the FGA score using the TIS and BBS scores, the correlation coefficient ( $r$ ) was 0.816 , the coefficient of determination $\left(R^{2}\right)$ was 0.666 , the regression constant was -8.864 , and the regression coefficient for the FGA score were 0.420 for TIS and 0.468 for BBS. In the group with an FGA score of $>15$ for regression equation for predicting FGA score using TIS and BBS score, the correlation coefficient ( $\mathrm{r}$ ) was 0.572 , the coefficient of determination $\left(\mathrm{R}^{2}\right)$ was 0.327 the regression
Table 5. Output for multiple regression analyses for the prediction of the FGA score from the TIS and BBS $(\mathrm{N}=68)$

\begin{tabular}{lllc}
\hline \multicolumn{1}{c}{ Clinical scale } & & \multicolumn{1}{c}{$\beta^{\mathrm{a}}$} & $p$-value \\
\hline FGA (scores) & TIS & 0.284 & 0.016 \\
& $\mathrm{BBS}$ & 0.575 & $<0.001$ \\
& \multicolumn{2}{c}{$\mathrm{R}^{2}=0.666, \mathrm{~F}=64.911, p=0.000$} \\
FGA $>15\left(\mathrm{n}_{1}=37\right)$ & $\mathrm{TIS}$ & 0.200 & 0.242 \\
& $\mathrm{BBS}$ & 0.437 & 0.014 \\
& $\mathrm{R}{ }^{2}=0.327, \mathrm{~F}=8.254, p=0.001$ \\
FGA $\leq 15\left(\mathrm{n}_{2}=31\right)$ & $\mathrm{TIS}$ & 0.067 & 0.761 \\
& $\mathrm{BBS}$ & 0.514 & 0.026 \\
& \multicolumn{2}{c}{$\mathrm{R}^{2}=0.316, \mathrm{~F}=6.478, p=0.005$} \\
\hline
\end{tabular}

FGA: Functional Gait Assessment, TIS: Trunk Impairment scale, BBS: Berg Balance scale.

${ }^{\mathrm{a}}$ Standardized coefficient $(\beta)$.

constant was 3.217, and the regression coefficient for the FGA score was 0.340 for BBS. In the group with an FGA score of $\leq 15$ for regression equation for predicting FGA score using TIS and BBS score, the correlation coefficient (r) was 0.562 , the coefficient of determination $\left(\mathrm{R}^{2}\right)$ was 0.316 the regression constant was 2.824 , and the regression coefficient for the FGA score was 0.199 for BBS. As seen in Table 5, the most significant variable that determined the FGA score in all the FGA groups were the TIS and BBS scores, but the BBS score was the most significant variable for determining the FGA scores for either group with an FGA of $>15$ or FGA $\leq 15$. The TIS was not a significant predictor of the FGA score in the FGA $>15$ group $(p=0.242)$ and FGA $\leq 15$ group $(p=0.761)$.

\section{Discussion}

This study aimed to predict balance and gait ability using BBS and FGS scores based on trunk performance, which were based on TIS scores, in persons with stroke. The results of this study showed that the TIS is valuable in predicting balance and walking ability in persons with stroke. In regards to balance based on BBS scores, the TIS has a $61 \%$ ability to predict the BBS score in stroke survivors, and es- 
pecially more predictable in the group with BBS scores of below $45(50 \%)$ and has a correlative $(\mathrm{r}=0.70)$ role in the persons with stroke rather than the group with a BBS score of below 45 of BBS. In regards to walking ability based on the FGA, the TIS has a $53.7 \%$ ability to predict FGA scores in the stroke survivors. However, it is not highly predictive in groups with FGS scores below or above 15 in stroke survivors. The BBS has a more predictive ability of $66.6 \%$ with the TIS for FGA score in stroke patients, however in the group below or above 15 of FGA is only had predictive ability of BBS for FGA score in stroke patients.

The trunk plays a critical role rather than the extremities during stroke rehabilitation, and is an essential component for developing coordinative movements of the extremities for balance as well as for motor tasks $[3,6]$. In this study, the TIS was used to predict balance and gait ability based on the clinical scales for stroke survivors. The TIS had a high correlation coefficient $(\mathrm{r}=0.781)$ and a predictive ability of $61 \%$ for the BBS score, and a high correlation coefficient ( $\mathrm{r}=$ 0.707 ) and a predictive ability of $50 \%$ for those with BBS scores below 45 rather than above. This result shows that balance ability is greatly correlated with TIS scores in persons with stroke. Having trunk control is critical in assuming an upright position and for weight shifting during static and dynamic postural control [3]. Verheyden et al. [2] pointed out that trunk function is more important in sitting balance and trunk stabilization is important for performing selective movement. In this study, the $<45$ BBS score group was more affected by the TIS score. Leddy et al. [17] suggested that the BBS score of 47 can discriminate a faller with maximized sensitivity and specificity. Karthikbabu et al. [3] mentioned that if trunk impairment is directly affected during upright position against gravity, there will be loss of stabilization of the trunk and will be compensated by increasing spasticity in the distal part. The BBS mostly consists of standing activities that require the upright position and movement of the trunk. Therefore, those with trunk impairment may exhibit poor BBS scores.

In the predictive FGA score by TIS had high correlation coefficient ( $\mathrm{r}=0.733$ ), but the predictive ability of TIS had a $53.7 \%$ for FGA that is relatively low rather than BBS. And also, TIS had low correlation coefficient and low predictive ability in each group of FGA below or above 15. Kim et al. [9] and Verheyden et al. [7] reported that TIS is related to gait ability, and also could use for clinical application for measuring gait ability with severity of trunk impairment. Efficiently walking is required rotations between shoulder and pelvic girdle, and these combinations will be supporting stabilization to more selective movement and other stable movement [2]. FGA is more dynamic items for postural adjustment during testing rather than BBS [17], and gait variability is more depend on balance control directly [11]. Therefore FGA is including more comprehensive information of balance and gait ability during testing rather than ABC, BBS, and TUG [12]. These reasons were reflected that TIS is a one of strong predictive ability, and if combined BBS and TIS had more predictive ability with $66.6 \%$ and correlation coefficient $(\mathrm{r}=0.816)$. However, in the group below 15 and above 15, TIS is not showed predictive ability, BBS is only showed predictive ability in each group of FGA. Because of FGA items had high variability, and FGA score of 20 is the criteria for identifying falling risk in older adults, and FGA score 15 for Parkinson disease $[12,17]$. In this study, 15 score of FGA score is used for dividing group with higher ability and lower ability, and the number of subjects is not too enough, and gait ability is more complicating requited dynamic balance, but BBS is mostly measured with static balance and TIS is measured with seating position. FGA are consisted with dynamic balance and more coordinative movement in stance and upright during walking. That is indicated that BBS is more predictive variable for FGA rather than TIS in stroke patients despite of considering group. The limitations of this study, there are not enough participants and TIS is not to cover all range of score in stroke patients. And also, it did not use of any kinds of motion analysis systems and other clinical scales for prediction in this study. Therefore, the results cannot be generalized to the all range of stroke patients.

In conclusion, this study aimed to predict balance and gait ability with trunk ability using clinical scales. The TIS, BBS, and FGA scores of 68 persons with stroke were used for analyses with regression. The results suggested that the TIS is the strongest variable for predicting BBS in stroke survivors with poor balance ability. However, the TIS was not enough to predict the FGA and BBS scores in those with higher balance ability. Also, the BBS and TIS can be used for predicting variables for FGA. Therefore, the use of predictive variable with TIS is not enough supporting gait ability. TIS is a strong predictive variable in stroke survivors with poor balance ability.

\section{Acknowledgements}

This work was supported by the 2017 Research-Year 
Grant of Jeonju University.

\section{Conflict of Interest}

The author declared no potential conflicts of interest with respect to the authorship and/or publication of this article.

\section{References}

1. Fujiwara T, Liu M, Tsuji T, Sonoda S, Mizuno K, Akaboshi K, et al. Development of a new measure to assess trunk impairment after stroke (trunk impairment Scale): its psychometric properties. Am J Phys Med Rehabil 2004;83:681-8.

2. Verheyden G, Nieuwboer A, Van de Winckel A, De Weerdt W. Clinical tools to measure trunk performance after stroke: a systematic review of the literature. Clin Rehabil 2007;21:387-94.

3. Karthikbabu S, Chakrapani M, Ganeshan S, Rakshith KC, Nafeez S, Prem V. A review on assessment and treatment of the trunk in stroke: a need or luxury. Neural Regen Res 2012;7: 1974-7.

4. Grimpampi E, Bonnet V, Taviani A, Mazzà C. Estimate of lower trunk angles in pathological gaits using gyroscope data. Gait Posture 2013;38:523-7.

5. Likhi M, Jidesh VV, Kanagaraj R, George JK. Does trunk, arm, or leg control correlate best with overall function in stroke subjects? Top Stroke Rehabil 2013;20:62-7.

6. Kim JH, Lee SM, Jeon SH. Correlations among trunk impairment, functional performance, and muscle activity during forward reaching tasks in patients with chronic stroke. J Phys Ther Sci 2015;27:2955-8.

7. Verheyden G, Vereeck L, Truijen S, Troch M, Herregodts I, Lafosse $\mathrm{C}$, et al. Trunk performance after stroke and the relationship with balance, gait and functional ability. Clin Rehabil 2006;20:451-8.

8. Jijimol G, Fayaz RK, Vijesh PV. Correlation of trunk impairment with balance in patients with chronic stroke. NeuroRehabilitation 2013;32:323-5.

9. Kim TJ, Seo KM, Kim DK, Kang SH. The relationship between initial trunk performances and functional prognosis in patients with stroke. Ann Rehabil Med 2015;39:66-73.

10. Shumway-Cook A, Woollacott MH. Clinical management of the patient with a postural control disorder. In: Shumway-Cook A, Woollacott $\mathrm{MH}$, editors. Motor control: translating research into clinical practice. 4th ed. Philadelphia: Lippomcptt Williams \& Wilkins; 2012. p. 257-96.

11. Mansfield A, Wong JS, McIlroy WE, Biasin L, Brunton K, Bayley M, et al. Do measures of reactive balance control predict falls in people with stroke returning to the community? Physiotherapy 2015;101:373-80.

12. Wrisley DM, Kumar NA. Functional gait assessment: concurrent, discriminative, and predictive validity in communitydwelling older adults. Phys Ther 2010;90:761-73.

13. Watkins LG, Portney MP. Regression. In: Watkins LG, Portney MP, editors. Foundations of clinical research: applications to practice. Upper Saddle River, NJ: Prentice Hall PTR; 1993. p. 457-84.

14. Di Monaco M, Trucco M, Di Monaco R, Tappero R, Cavanna A. The relationship between initial trunk control or postural balance and inpatient rehabilitation outcome after stroke: a prospective comparative study. Clin Rehabil 2010;24:543-54.

15. Verheyden G, Nieuwboer A, Mertin J, Preger R, Kiekens C, De Weerdt W. The trunk impairment scale: a new tool to measure motor impairment of the trunk after stroke. Clin Rehabil 2004; 18:326-34.

16. Verheyden G, Nieuwboer A, Feys H, Thijs V, Vaes K, De Weerdt W. Discriminant ability of the trunk impairment scale: a comparison between stroke patients and healthy individuals. Disabil Rehabil 2005;27:1023-8.

17. Leddy AL, Crowner BE, Earhart GM. Functional gait assessment and balance evaluation system test: reliability, validity, sensitivity, and specificity for identifying individuals with Parkinson disease who fall. Phys Ther 2011;91:102-13.

18. Berg KO, Wood-Dauphinee SL, Williams JI, Maki B. Measuring balance in the elderly: validation of an instrument. Can J Public Health 1992;83 Suppl 2:S7-11.

19. Berg K, Wood-Dauphinee S, Williams JI. The balance scale: reliability assessment with elderly residents and patients with an acute stroke. Scand J Rehabil Med 1995;27:27-36.

20. Blum L, Korner-Bitensky N. Usefulness of the Berg Balance scale in stroke rehabilitation: a systematic review. Phys Ther 2008;88:559-66.

21. Lin JH, Hsu MJ, Hsu HW, Wu HC, Hsieh CL. Psychometric comparisons of 3 functional ambulation measures for patients with stroke. Stroke 2010;41:2021-5.

22. Wrisley DM, Marchetti GF, Kuharsky DK, Whitney SL. Reliability, internal consistency, and validity of data obtained with the functional gait assessment. Phys Ther 2004;84:906-18. 